\title{
A LYAPUNOV INEQUALITY AND FORCED OSCILLATIONS IN GENERAL NONLINEAR $n$ TH ORDER DIFFERENTIAL-DIFFERENCE EQUATIONS $\dagger$
}

\author{
by LU-SAN CHEN
}

(Received 9 March, 1976)

1. Introduction. The purpose of this paper is to consider the general nonlinear $n$th order differential-difference equation

$$
\left.\left[r(t) h\left(y^{\prime}(t)\right)\right]^{(n-1)}+a(t) y(t) f(y(t-\tau(t)))=b, t\right)
$$

and derive an inequality of Lyapunov type. Later we use this inequality to find conditions to ensure that the oscillatory solutions of equation (1) tend to zero as $t \rightarrow \infty$. The conditions that ensure that the oscillatory solutions of equation (1) tend to zero, also cause all solutions of equation

to be non-oscillatory.

$$
\left[r(t) h\left(y^{\prime}(t)\right)\right]^{(n-1)}+a(t) y(t) f(y(t-\tau(t)))=0
$$

The classical Lyapunov inequality states that if $y(t)$ is a non-trivial solution of the second order linear equation

$$
y^{\prime \prime}(t)+a(t) y(t)=0,
$$

where $a(t)$ is real and continuous, and if $y(t)$ vanishes at least twice on the interval $\left[t_{1}, t_{2}\right]$, then

$$
\left(t_{2}-t_{1}\right) \int_{t_{1}}^{t_{2}} a^{+}(t) d t>4 \text {, where } a^{+}(t)=\max (a(t), 0) \text {. }
$$

This inequality is well known to be the sharpest possible, so that 4 cannot be replaced by larger constant, cf. [1]. In general, this inequality is not true for delay equations. As an example, the equation

$$
y^{\prime \prime}(t)-y(t-\pi)=0
$$

has as a nontrivial solution $y(t)=\sin t$ on $(0, \infty)$ subject to $y(t)=\sin t, t \in[-\pi, 0]$, but taking $t_{1}=0, t_{2}=\pi, a(t)=-1$, we find that the conclusion of the inequality is not true.

Eliason [2] considered the equation

$$
\left[r(t) y^{\prime}(t)\right]^{\prime}+a(t) y(t) f(y(t))=0
$$

and proved a more general version of Lyapunov inequality. Recently, Dahiya-Singh [4] considered the equation

$$
\left[r(t) h\left(y^{\prime}(t)\right)\right]^{\prime}+a(t) y(t) f(y(t-\tau(t)))=0,
$$

and, more recently, Singh [5] also considered the equation

$$
\left[r(t) y^{\prime}(t)\right]^{(n-1)}+a(t) y(t)=f(t)
$$

and proved an extension of this inequality which is a particular case of our result.

† This research was supported,by the National Science Council.

Glasgow Math. J. 18 (1977) 161-166.

$\mathbf{L}$ 
We shall consider only those solutions of the equation (1) which exist on some half-line $\left[t_{\xi}, \infty\right)$, where $t_{\xi}$ may depend on the particular solution, and are nontrivial in any neighbourhood of infinity. Such a solution is called oscillatory if it has arbitrarily large zeros; otherwise it is called non-oscillatory. In addition the following assumptions will be made for the rest of this paper.

Assumptions. (i) $a(t)$ and $b(t)$ are continuous real-valued functions on $[0, \infty)$.

(ii) $r(t)$ is a continuous and positive real-valued function on $[0, \infty)$.

(iii) $\tau(t)$ is continuous positive and bounded so that there exists some positive constant $m$ such that $0<\tau(t) \leqq m$.

(iv) $h(x)$ is continuously differentiable on $(-\infty, \infty)$ and is an odd function such that $\operatorname{sgn} h(x)=\operatorname{sgn} x$; there exists $\beta>0$ such that $0<x / h(x) \leqq \beta$, and $\lim _{x \rightarrow 0}(x / h(x))$ exists finitely so
that $x / h(x)$ is continuously differentiable on $[0, \infty)$.

(v) $f(x)$ is a continuous, even, real positive function on $(-\infty, \infty)$ and increasing on $[0, \infty)$ with $f(0)=0$.

To obtain our results we make use of the following lemma adapted from Singh [5].

Lemma. Assume that $\alpha_{1}>\alpha_{2}>\alpha_{3}>\ldots>\alpha_{n-2}$ are, respectively, zeros of

$$
\left[r(t) h\left(y^{\prime}(t)\right)\right]^{\prime}, \quad\left[r(t) h\left(y^{\prime}(t)\right)\right]^{\prime \prime}, \quad \ldots, \quad\left[r(t) h\left(y^{\prime}(t)\right)\right]^{(n-3)}, \quad\left[r(t) h\left(y^{\prime}(t)\right)\right]^{(n-2)},
$$

where $y(t)$ is a solution of equation (1). Furthermore, suppose that $t_{1}<\alpha_{n-2}$ and $t_{2}>\alpha_{1}$ are zeros of $y(t)$. Let

Then

$$
L=\sup \left\{y(t): t \in\left(t_{1}-m, t_{2}\right), t_{1}, t_{2}>m\right\} \text { and } M=\sup \left\{|y(t)|: t \in\left[t_{1}, t_{2}\right]\right\} .
$$

$$
4 \leqq \beta \int_{t_{1}}^{t_{2}} \frac{d t}{r(t)}\left\{f(L) \int_{t_{1}}^{t_{2}} \frac{\left(t-t_{1}\right)^{n-2}}{(n-2) !}|a(t)| d t+\frac{1}{M} \int_{t_{1}}^{t_{2}} \frac{\left(t-t_{1}\right)^{n-2}}{(n-2) !}|b(t)| d t\right\} .
$$

Proof. Integration of (1) $n-2$ times gives

$$
\begin{aligned}
(-1)^{n}\left[r(t) h\left(y^{\prime}(t)\right)\right]^{\prime}+\int_{t}^{\alpha_{1}} \int_{s_{2}}^{\alpha_{2}} \ldots \int_{s_{n-2}}^{\alpha_{n-2}} a(s) y(s) f(y(s-\tau(s))) d s d s_{n-2} \ldots d s_{2} \\
\quad=\int_{t}^{\alpha_{1}} \int_{s_{2}}^{\alpha_{2}} \ldots \int_{s_{n-2}}^{\alpha_{n-2}} b(s) d s d s_{n-2} \ldots d s_{2} .
\end{aligned}
$$

Since $\alpha_{1}>\alpha_{2}>\alpha_{3}>\ldots>\alpha_{n-2}$, we obtain from (4),

$$
\begin{aligned}
\left|\left[r(t) h\left(y^{\prime}(t)\right)\right]^{\prime}\right| \leqq \int_{t}^{\alpha_{1}} \int_{s_{2}}^{\alpha_{1}} \ldots \int_{s_{n-2}}^{\alpha_{1}}|a(s)\|y(s)\| f(y(s-\tau(s)))| d s d s_{n-2} \ldots d s_{2} \\
\quad+\int_{t}^{\alpha_{1}} \int_{s_{2}}^{\alpha_{1}} \ldots \int_{s_{n-2}}^{\alpha_{1}}|b(s)| d s d s_{n-2} \ldots d s_{2},
\end{aligned}
$$

which implies

$$
\left|\left[r(t) h\left(y^{\prime}(t)\right)\right]^{\prime}\right| \leqq \int_{t}^{\alpha_{1}} \frac{(s-t)^{n-3}}{(n-3) !}|a(s)\|y(s)\| f(y(s-\tau(s)))| d s+\int_{t}^{\alpha_{1}} \frac{(s-t)^{n-3}}{(n-3) !}|b(s)| d s .
$$


Let

$$
M=\left|y\left(t_{0}\right)\right|, \quad t_{0} \in\left[t_{1}, t_{2}\right]
$$

Now

$$
\pm M=y\left(t_{0}\right)=\int_{t_{1}}^{t_{0}} y^{\prime}(t) d t
$$

which implies

$$
M \leqq \int_{t_{1}}^{t_{0}}\left|y^{\prime}(t)\right| d t
$$

Similarly

$$
M \leqq \int_{t_{0}}^{t_{2}}\left|y^{\prime}(t)\right| d t
$$

From (7) and (8),

$$
2 M \leqq \int_{t_{1}}^{t_{2}}\left|y^{\prime}(t)\right| d t
$$

By Schwarz's inequality, we get

$$
4 M^{2} \leqq \int_{t_{1}}^{t_{2}} \frac{y^{\prime}(t)}{h\left(y^{\prime}(t)\right)} \frac{d t}{r(t)} \int_{t_{1}}^{t_{2}}\left[r(t) h\left(y^{\prime}(t)\right)\right] y^{\prime}(t) d t,
$$

since $y^{\prime}(t) / h\left(y^{\prime}(t)\right)$ is continuous and positive. Therefore

$$
4 M^{2} \leqq \beta \int_{t_{1}}^{t_{2}} \frac{d t}{r(t)} \int_{t_{1}}^{t_{2}}\left[r(t) h\left(y^{\prime}(t)\right)\right] y^{\prime}(t) d t,
$$

since $0<y^{\prime}(t) / h\left(y^{\prime}(t)\right) \leqq \beta$.

Integrating the second integral of the right-hand side by parts, we have

$$
\frac{4 M^{2}}{\beta \int_{t_{1}}^{t_{2}} \frac{d t}{r(t)}} \leqq-\int_{t_{1}}^{t_{2}} y(t)\left[r(t) h\left(y^{\prime}(t)\right)\right]^{\prime} d t,
$$

since $y\left(t_{1}\right)=y\left(t_{2}\right)=0$. It follows, from (10), that

$$
\frac{4 M^{2}}{\beta \int_{t_{1}}^{t_{2}} \frac{d t}{r(t)}} \leqq \int_{t_{1}}^{t_{2}}\left|y(t) \|\left[r(t) h\left(y^{\prime}(t)\right)\right]^{\prime}\right| d t
$$

From (6) and (11),

$$
\frac{4 M}{\beta \int_{t_{1}}^{t_{2}} \frac{d t}{r(t)}} \leqq \int_{t_{1}}^{t_{2}}\left|\left[r(t) h\left(y^{\prime}(t)\right)\right]^{\prime}\right| d t
$$


From (5) and (12), we have

$$
\begin{aligned}
\frac{4 M}{\beta \int_{t_{1}}^{t_{2}} \frac{d t}{r(t)}} \leqq & \int_{t_{1}}^{t_{2}} \int_{s}^{\alpha_{1}} \frac{(x-s)^{n-3}}{(n-3) !}|a(x) \| y(x)| f(L) d x d s \\
& +\int_{t_{1}}^{t_{2}} \int_{s}^{\alpha_{1}} \frac{(x-s)^{n-3}}{(n-3) !}|b(x)| d x d s .
\end{aligned}
$$

Dividing by $M$ and noting that $t_{2}>\alpha_{1}$, we have, from (13),

From (14), we have

$$
\begin{aligned}
\frac{4}{\beta \int_{t_{1}}^{t_{2}} \frac{d t}{r(t)}} \leqq & f(L) \int_{t_{1}}^{t_{2}} \int_{s}^{t_{2}} \frac{(x-s)^{n-3}}{(n-3) !}|a(x)| d x d s \\
& +\frac{1}{M} \int_{t_{1}}^{t_{2}} \int_{s}^{t_{2}} \frac{(x-s)^{n-3}}{(n-3) !}|b(x)| d x d s .
\end{aligned}
$$

$$
4 \leqq \beta \int_{t_{1}}^{t_{2}} \frac{d t}{r(t)}\left\{f(L) \int_{t_{1}}^{t_{2}} \frac{\left(s-t_{1}\right)^{n-2}}{(n-2) !}|a(s)| d s+\frac{1}{M} \int_{t_{1}}^{t_{2}} \frac{\left(s-t_{1}\right)^{n-2}}{(n-2) !}|b(s)| d s\right\},
$$

and the proof is complete.

Remark 1. Eliason [2] has discussed the lemma in the case $n=2, h\left(y^{\prime}(t)\right) \equiv y^{\prime}(t)$, $b(t) \equiv 0$ and $f(y(t-\tau(t))) \equiv f(y(t))$. Dahiya-Singh [4] has discussed it in the case $n=2$, $b(t) \equiv 0$, and Singh [5] also has discussed it in the case $r(t) \equiv 1, b(t) \equiv 0, h\left(y^{\prime}(t)\right) \equiv y^{\prime}(t)$ and $f(y(t-\tau(t))) \equiv 1$.

2. Theorems. We now give a generalization of Lyapunov inequality for the equation

$$
y^{(n)}(t)+a(t) y(t)=0 .
$$

THEOREM 1. Assume that $r_{2}>r_{3}>\ldots>r_{n-1}$ are zeros of $y^{\prime \prime}(t), y^{\prime \prime \prime}(t), \ldots, y^{(n-1)}(t)$ respectively, where $y(t)$ is a solution of equation (15). Let $t_{1}<r_{n-1}$ and $t_{2}>r_{2}$ be zeros of $y(t)$. Then

$$
\frac{4}{t_{2}-t_{1}} \leqq \int_{t_{1}}^{t_{2}} \frac{\left(t-t_{1}\right)^{n-2}}{(n-2) !}|a(t)| d t .
$$

Proof. In the lemma, we put

$$
r(t) \equiv 1, \quad h\left(y^{\prime}(t)\right) \equiv y^{\prime}(t), \quad f(y(t-\tau(t))) \equiv 1 \quad \text { and } \quad b(t) \equiv 0,
$$

and the conclusion follows.

TheOREM 2. Assume that $f(x)$ is bounded and

$$
\int^{\infty} t^{n-2}|a(t)| d t<\infty
$$




$$
\int^{\infty} t^{n-2}|b(t)| d t<\infty
$$

and

$$
\int^{\infty} \frac{d t}{r(t)}<\infty .
$$

Let $y(t)$ be an oscillatory solution of equation (1). Then

$$
\lim _{t \rightarrow \infty} y(t)=0 \text {. }
$$

Proof. Let

$$
M_{1}=\sup _{0 \leqq x<\infty} f(x) .
$$

Suppose to the contrary that $\lim _{t \rightarrow \infty} y(t) \neq 0$. Then

and for some positive $d$,

$$
\liminf _{t \rightarrow \infty}|y(t)|=0
$$

$$
\limsup _{t \rightarrow \infty}|y(t)|>2 d \text {. }
$$

Due to the oscillatory nature of $y(t),\left[r(t) h\left(y^{\prime}(t)\right)\right]^{(n-2)}$ must be oscillatory. In fact if $\left[r(t) h\left(y^{\prime}(t)\right)\right]^{(n-2)}$ is non-oscillatory, then $r(t) h\left(y^{\prime}(t)\right)$ assumes one sign eventually. Since $r(t)>0$, $\operatorname{sgn} h\left(y^{\prime}(t)\right)=\operatorname{sgn} y^{\prime}(t), h\left(y^{\prime}(t)\right)$ is continuous and odd, $y^{\prime}(t)$ becomes non-oscillatory which in turn forces $y(t)$ to be non-oscillatory, which is a contradiction. Hence $\left[r(t) h\left(y^{\prime}(t)\right)\right]^{(n-2)}$ is oscillatory. Similarly

$$
\left[r(t) h\left(y^{\prime}(t)\right)\right]^{(n-3)},\left[r(t) h\left(y^{\prime}(t)\right)\right]^{(n-4)}, \ldots,\left[r(t) h\left(y^{\prime}(t)\right)\right]^{\prime}
$$

are all oscillatory. Let $T$ be large enough so that

$$
\begin{gathered}
\int_{T}^{\infty} t^{n-2}|a(t)| d t<\frac{1}{M_{1}} \\
\int_{T}^{\infty} t^{n-2}|b(t)| d t<d
\end{gathered}
$$

and

$$
\int_{T}^{\infty} \frac{d t}{r(t)}<\frac{1}{\beta}
$$

Let $T<t_{1}<\alpha_{n-2}<\ldots<\alpha_{3}<\alpha_{2}<\alpha_{1}<T_{0}$ be points such that

and

$$
\begin{gathered}
y\left(t_{1}\right)=0, \\
{\left[r\left(\alpha_{i}\right) h\left(y^{\prime}\left(\alpha_{i}\right)\right)\right]^{(i)}=0, \quad i=1,2, \ldots, n-2,}
\end{gathered}
$$

$$
M=\sup _{t ı t \leqq T_{0}}|y(t)|>d .
$$


Let $t_{2}>T_{0}$ be another zero of $y(t)$. Let

$$
M_{0}=\sup _{t_{1} \leqq t \leqq t_{2}}|y(t)| .
$$

Then $M_{0}>d$. From the conclusion of the lemma, we have

$$
4 \leqq \beta \int_{t_{1}}^{t_{2}} \frac{d t}{r(t)}\left\{M_{1} \int_{t_{1}}^{t_{2}} \frac{\left(s-t_{1}\right)^{n-2}}{(n-2) !}|a(s)| d s+\frac{1}{M_{0}} \int_{t_{1}}^{t_{2}} \frac{\left(s-t_{1}\right)^{n-2}}{(n-2) !}|b(s)| d s\right\} .
$$

From (23), (24), (25), the fact that $M_{0}>d$ and (29), we have

$$
4 \leqq 1+(d / d)=2 \text {. }
$$

This contradiction proves the theorem.

RemaRK 2. For the case $h\left(y^{\prime}(t)\right) \equiv y^{\prime}(t), f(y(t-\tau(t))) \equiv 1$, our Theorem 2 coincides with Theorem 1 of Singh [5].

THEOREM 3. Suppose that (17) and (19) are satisfied, and that $f(x)$ is bounded. Then every solution of (2) is non-oscillatory.

Proof. Following the proof of Theorem 2, we arrive at conclusion (29). From (29), we get

$$
4 \leqq \beta \int_{t_{1}}^{t_{2}} \frac{d t}{r(t)}\left\{M_{1} \int_{t_{1}}^{t_{2}} \frac{\left(s-t_{1}\right)^{n-2}}{(n-2) !}|a(s)| d s\right\} \leqq 1,
$$

using (23) and (25). This contradiction proves the theorem.

The author wishes to express his thanks to the referee for his helpful suggestions.

\section{REFERENCES}

1. P. Hartman, Ordinary differential equations (Wiley, 1964), 345-346, 401.

2. S. B. Eliason, A Lyapunov inequality for a certain second order nonlinear differential equation, J. London Math. Soc. (2) 2 (1970), 461-466.

3. J. S. Bradley, Oscillation theorems for a second order delay equation, J. Differential Equations 8 (1970), 397-403.

4. R. S. Dahiya and B. Singh, A Lyapunov inequality and nonoscillation theorem for a second order nonlinear differential-difference equation, J. Mathematical and Physical Sci. 7 (1973), 163-170.

5. B. Singh, Forced oscillations in general ordinary differential equations, Tamkang J. Math. 6 (1975), 5-11.

6. M. E. Hammelt, Nonoscillation properties of a nonlinear differential equation, Proc. Amer. Math. Soc. 30 (1971), 92-96.

7. S. Londen, Some nonoscillation theorems for a second order nonlinear differential equation, SIAM J. Math. Anal. 4 (1973), 460-465.

Department of Mathematics

National Central University

CHUNG-LI

TAIWAN 